\title{
Noncommutative Field Theory
}

\author{
M. Gomes \\ Instituto de Física da Universidade de São Paulo \\ Caixa Postal 66318, 05315-970, São Paulo, SP, Brazil
}

Received on 9 March, 2002

\begin{abstract}
Noncommutative field theories present many surprising properties. As a consequence of the noncommutativity, high momentum modes do not decouple from the physics at large distances leading to the appearance of infrared singularities even in theories without massless particles. Being nonintegrable, these infrared singularities destroy the usual perturbative expansions. We discuss talk alternative procedures to, we examine control this situation. In the low momentum regime of the Wess-Zumino model the effect of the underlying nonlocality on the nonrelativistic potential will be examined and some physical implications.
\end{abstract}

It is a relatively old question if there exists or not a minimum length beyond which no strict localization is possible. In the case of a positive answer, interactions would have to be smeared over the minimum length, possibly eliminating the ultraviolet divergences of the perturbative series. Such an idea was put forward in the forties of the last century [1] but never achieved popularity, mainly because of the success of the renormalization program for QED. More recently, it has been argued that for very small distances, distances of the order of the Planck's length $\left(10^{-33} \mathrm{~cm}\right)$, the measurement of coordinates is meaningless due to the appearance of strong gravitational fields which will prevent everything to transmit information [2]. According to the Heisenberg uncertainty relations, a precise measurement of a coordinate leads to a very high indeterminacy in the momentum. In this process, therefore, a great amount of energy is transmitted to the observed system and the corresponding energy-momentum tensor generates a strong gravitational field, as predicted by the Einstein's equation. The more precise the measurement of the coordinates the bigger will be the gravitational coming from the referred measurement. Whenever this field is so strong as to prevent light or other signs to come out from the region under observation no operational meaning can be given to the idea of strict localization.

From a quantum mechanical point of view, the above analysis suggest the validity of the following position uncertainty relations

$$
\Delta q^{\mu} \Delta q^{\nu} \approx\left|\Theta^{\mu \nu}\right|
$$

where $\Theta^{\mu \nu}$ is an antisymmetric matrix whose elements are of the order of the square of the minimum length. In fact, by postulating (1) it follows that, if one coordinate is precisely measured, there are high indeterminacies in the remaining ones.

The possibility unveiled by (1) is a direct consequence of

$$
\left[q^{\mu}, q^{\nu}\right]=i \Theta^{\mu \nu}
$$

so that classical fields become noncommutative operators after the quantization prescribed in (2).

Noncommutative quantum field theories have also arised in the context of string theory as it has been shown that the dynamics of a D-brane in the presence of an antisymmetric field can be described, in certain limits, in terms of a gauge theory deformed by a Moyal product [3]; this product is a characteristic of the noncommutativity. In this meeting, the strings aspects of noncommutative space have been discussed in the Prof. Matsuo talk. Here, I only would like to remark that the discovery of noncommutative field theory as a certain limit of string theory raised the hopes that this limit should lead to a consistent theory (see $[4,5]$ for recent reviews). I will discuss this aspect and will stress that this is I highly nontrivial point.

Leaving aside questions posed by the loss of Lorentz invariance, a first observation is that a violation of causality and unitarity occurs when the noncommutativity involves the time coordinate $[6,7]$.

The next observation concerns the entanglement of scales: small distances in one direction imply large distances in the other, as follows from (1). Usually, the ultraviolet behavior of a field theory is unrelated to the infrared one. Thus, in massive $\phi^{4}$ there are quadratic and logarithmic ultraviolet divergences coexisting with infrared finiteness. In the noncommutative case matters are more subtle. The simplest example where the the so called IR/UV (infrared/ultraviolet) mixing occurs is provided by the one-loop seagull graph whose 
noncommutative amplitude is given by

$$
\int d^{4} k \frac{\cos \left(k_{\mu} \Theta^{\mu \nu} p_{\nu}\right)}{k^{2}-m^{2}} \text {. }
$$

The appearance of trigonometric factors is a hallmark of the noncommutative situation. It provides an oscillating factor which effectively damps the ultraviolet behavior. The integral turns out to be finite but, in a way different from the usual situation, it depends on the external momentum. If this momentum were zero (or if $\Theta$ were zero) the integral would be quadratically divergent. We are thus led to the conclusion that for small $p$ the integral should behave as

$$
\approx \frac{1}{p^{2} \Theta^{2}}
$$

When multiple insertions of this graph are made into a large diagram they generate non integrable infrared singularities. This feature produces the breakdown of the perturbative scheme in many of the renormalizable theories. We shall return to this point later on.

We now proceed to describe some of the steps to be followed to build some simple field theories in noncommutative space. First of all, field operators may be constructed via the so called Weyl-Moyal correspondence. To this end we introduce the operator

$$
T(k)=\mathrm{e}^{i k_{\mu} q^{\mu}},
$$

which obeys the rules [8]:

1. $T^{\dagger}(k)=T(-k)$ if $q^{\mu^{\dagger}}=q^{\mu}$.

2. $T(k) T\left(k^{\prime}\right)=T\left(k+k^{\prime}\right) \mathrm{e}^{-\frac{i}{2} k_{\mu} k_{\nu}^{\prime} \Theta^{\mu \nu}}$ which follows immediately after the application of the BakerHausdorf formula

$$
\mathrm{e}^{A} \mathrm{e}^{B}=\mathrm{e}^{A+B} \mathrm{e}^{\frac{1}{2}[A, B]},
$$

for $[A, B]=\mathrm{c}$ number.

$$
\text { 3. } \operatorname{Tr} T(k)=(2 \pi)^{n} \prod_{\mu} \delta\left(k_{\mu}\right)
$$

The operators $T(k)$ will be assumed to form a basis in the space of the field operators in analogous way as $e^{i k x}$ form a basis for squared integrable functions on the ordinary space. More precisely, to every sufficient smooth classical function $\phi(x)$ we associate the field operator (for notational simplicity $d x \equiv d^{n} x$ and $\left.d k \equiv d^{n} k\right)$

$$
\Phi=\frac{1}{(2 \pi)^{n}} \int d x d k T(k) \mathrm{e}^{i k_{\mu} x^{\mu}} \phi(x),
$$

or

$$
\Phi=\int \frac{d k}{(2 \pi)^{n}} T(k) \tilde{\phi}(k),
$$

in terms of the Fourier transform $\tilde{\phi}(k)$ of $\phi(x)$,

$$
\tilde{\phi}(k)=\int d x \mathrm{e}^{i k x} \phi(x) .
$$

We may verify that

$$
\phi(x)=\int \frac{d k}{(2 \pi)^{n}} e^{-i k x} \operatorname{Tr}\left[\Phi T^{\dagger}(k)\right] .
$$

This formula relates in a definite way a classical function $\phi(x)$ to a given operator $\Phi(q)$. It can be used to construct the so called Moyal product of the classical functions $\phi_{1}$ and $\phi_{2}$, corresponding to the product of the operators $\Phi_{1}$ and $\Phi_{2}$ which are associated to them:

$$
\phi_{1}(x) * \phi_{2}(x)=\int \frac{d k}{(2 \pi)^{n}} \mathrm{e}^{-i k x} \operatorname{Tr}\left[\Phi_{1} \Phi_{2} T^{\dagger}(k)\right] .
$$

The Moyal product is a highly nonlocal function involving an arbitrary number of derivatives. In fact, from the definition (11) it is possible to show that

$$
\phi_{1}(x) * \phi_{2}(x)=\lim _{y \rightarrow x} \mathrm{e}^{\frac{i}{2} \Theta^{\mu \nu} \frac{\partial}{\partial y^{\mu}} \frac{\partial}{\partial x^{\nu}}} \phi_{1}(y) \phi_{2}(x)=\phi_{1}(x) \phi_{2}(x)+\frac{i}{2} \Theta^{\mu \nu}\left(\partial_{\mu} \phi_{1}(x)\right)\left(\partial_{\nu} \phi_{2}(x)\right)+\ldots,
$$

which, due to the antisymmetry of $\Theta_{\mu \nu}$, differs from the ordinary pointwise product just by a total derivative. The same does not happen for the Moyal product of three or more fields. Employing the simplified notation $k_{i} \wedge k_{j} \equiv \frac{1}{2} k_{i}^{\mu} k_{j}^{\nu} \Theta_{\mu \nu}$, one finds

$$
\int d x \phi_{1}(x) * \phi_{2}(x) * \phi_{3}(x)=\int \frac{d k_{1}}{(2 \pi)^{n}} \frac{d k_{2}}{(2 \pi)^{n}} \frac{d k_{3}}{(2 \pi)^{n}}(2 \pi)^{n} \delta\left(k_{1}+k_{2}+k_{3}\right) \mathrm{e}^{-i k_{1} \wedge k_{2}} \tilde{\phi}_{1}\left(k_{1}\right) \tilde{\phi}_{2}\left(k_{2}\right) \tilde{\phi}_{3}\left(k_{3}\right)
$$

and, more generally, [8]

$$
\int d x \phi_{1}(x) \star \phi_{2}(x) \star \ldots \star \phi_{N}(x)=\int \prod \frac{d k_{i}}{(2 \pi)^{n}}(2 \pi)^{n} \delta\left(k_{1}+k_{2}+\ldots+k_{N}\right) \tilde{\phi}_{1}\left(k_{1}\right) \tilde{\phi}_{2}\left(k_{2}\right) \ldots \tilde{\phi}_{N}\left(k_{N}\right) \exp \left(-i \sum_{i<j} k_{i} \wedge k_{j}\right)
$$


As seen from the above expressions, the Feynman rules for a noncommutative field theory are similar to the ones in the commutative case, unless by oscillating trigonometric factors at the vertices. Actually, a special consideration is needed in the case of gauge symmetry: due to the noncommutativity, even in the $U(1)$ case one should start with a nonabelian expression for the field strength and then replace the ordinary product by the Moyal one[9, 10, 11].

The presence of the oscillating factors at the vertices regularize some of the ultraviolet divergences but produce infrared divergences. In such situation, we may envisage two procedures to go ahead: resummation and extension of the model. In the first approach the free propagator is modified by incorporating some part of the interaction to ameliorate the infrared behavior $[12,13]$. This modification leads to dispersion relations different from the usual ones and has some interesting physical implications in gauge theories [14]. However, there is a troublesome aspect of this method, namely, the absence of a clearly identifiable perturbative parameter. A second possibility which does not have this drawback consists in the addition of new interactions in such way improve the ultraviolet/infrared behavior of the amplitudes. In this respect supersymmetric theories appear as natural candidates. Actu- ally, it has been proved that the noncommutative WessZumino model and the $2+1$ dimensional nonlinear noncommutative sigma model are free from the dangerous IR/UV mixing [15, 16].

Besides the renormalization problems commented before one is naturally also interested in uncovering the physical content of a given noncommutative field theory. Here, one finds unusual and perhaps surprising aspects. For example, contrarily to its commutative counterpart, noncommutative QED exhibits asymptotic freedom. Also, the realization of spontaneous symmetry breakdown a necessary ingredient of a noncommutative "standard" model seems to depend on the nature of the underlying interaction. For the linear sigma model, it has been demonstrated that, at least up to one loop, spontaneous breakdown may occur for the $U(N)$ but not for the $O(N)$ symmetry if $N>2$ [17].

Following the thread delineated above, I would like to present some recent results on the low energy limit of the noncommutative Wess-Zumino model [18]. Starting from fermion-fermion and boson-boson scattering amplitudes, one may determine the equivalent potentials which fixes the non-relativistic dynamics. They are nonlocal potential with peculiar properties.

The Lagrangian describing the model is [19]

$$
\begin{aligned}
\mathcal{L}= & \frac{1}{2} A\left(-\partial^{2}\right) A+\frac{1}{2} B\left(-\partial^{2}\right) B+\frac{1}{2} \bar{\psi}(i \not \partial-m) \psi+\frac{1}{2} F^{2}+\frac{1}{2} G^{2}+m F A+m G B \\
& +g\left(F \star A \star A-F \star B \star B+G \star A \star B+G \star B \star A-\bar{\psi} \star \psi \star A-\bar{\psi} \star i \gamma_{5} \psi \star B\right) .
\end{aligned}
$$

where $A$ is a scalar field, $B$ is a pseudo scalar field, $\psi$ is a Majorana spinor field and $F$ and $G$ are, respectively, scalar and pseudoscalar auxiliary fields. Notice that composites bilinear in the basic fields are defined using ordinary, i. e., not Moyal ordered products.

Consider the scattering of two Majorana fermions. Let us designate by $p_{1}, p_{2}\left(p_{1}^{\prime}, p_{2}^{\prime}\right)$ and by $\epsilon_{1}, \epsilon_{2}\left(\epsilon_{1}^{\prime}, \epsilon_{2}^{\prime}\right)$ the four momenta and z-spin components of the incoming (outgoing) particles, respectively. We work in center of mass frame where the kinematics becomes simpler and one has that $p_{1}=(\omega, \vec{p}), p_{2}=(\omega,-\vec{p}), p_{1}^{\prime}=\left(\omega, \vec{p}^{\prime}\right)$, $p_{2}^{\prime}=\left(\omega,-\vec{p}^{\prime}\right),\left|\vec{p}^{\prime}\right|=|\vec{p}|$, and $\omega=\omega(\vec{p})=\sqrt{\vec{p}^{2}+m^{2}}$.

In the lowest order of perturbation there are three contributing amplitudes associated to the direct, exchange and "annihilation" channels (because we are using Majorana fields). The total amplitude is

$$
R==(2 \pi)^{4} \delta^{(4)}\left(p_{1}^{\prime}+p_{2}^{\prime}-p_{1}-p_{2}\right)\left(T_{a}+T_{b}+T_{c}\right)
$$

where in the nonrelativistic limit one finds

$$
\begin{aligned}
T_{a}^{L} & =-\frac{1}{(2 \pi)^{4}}\left(\frac{g}{\pi m}\right)^{2} \delta_{\epsilon_{1}^{\prime} \epsilon_{1}} \delta_{\epsilon_{2}^{\prime} \epsilon_{2}}\left[\frac{1}{2} \cos \left(m \Theta_{0 j} k^{j}\right)+\frac{1}{2} \cos \left(p^{i} \Theta_{i j} k^{j}\right)\right] \\
T_{b}^{L} & =\frac{1}{(2 \pi)^{4}}\left(\frac{g}{\pi m}\right)^{2} \delta_{\epsilon_{1}^{\prime} \epsilon_{2}} \delta_{\epsilon_{2}^{\prime} \epsilon_{1}}\left[\frac{1}{2} \cos \left(m \Theta_{0 j} k^{\prime j}\right)+\frac{1}{2} \cos \left(p^{i} \Theta_{i j} k^{\prime j}\right)\right]
\end{aligned}
$$




$$
\begin{aligned}
T_{c}^{L}= & \frac{1}{3(2 \pi)^{4}}\left(\frac{g}{\pi m}\right)^{2}\left\{\delta_{\epsilon_{1}^{\prime} \epsilon_{1}} \delta_{\epsilon_{2}^{\prime} \epsilon_{2}} \cos \left(m \Theta_{0 j} p^{j}\right) \cos \left[m \Theta_{0 j}\left(p^{j}-k^{j}\right)\right]\right. \\
& \left.-\delta_{\epsilon_{1}^{\prime} \epsilon_{2}} \delta_{\epsilon_{2}^{\prime} \epsilon_{1}} \cos \left(m \Theta_{0 j} p^{j}\right) \cos \left[m \Theta_{0 j}\left(p^{j}-k^{\prime j}\right)\right]\right\}
\end{aligned}
$$

where $k^{j} \equiv p^{j}-p^{\prime}{ }^{j}\left(k^{\prime} \equiv p^{j}+p^{j}\right)$ denotes the momentum transferred in the direct (exchange) scattering while the superscript $L$ signalizes that the above expressions only hold true for the low energy regime.

Concerning the boson-boson scattering, a straightforward calculation shows that the amplitudes corresponding to the direct and exchange processes become negligible in the low energy regime, the total amplitude being just

$$
\begin{aligned}
T^{L}= & \bar{T}_{c}^{L}=\frac{1}{6(2 \pi)^{4}}\left(\frac{g}{\pi m}\right)^{2} \cos \left(m \Theta_{0 j} p^{j}\right) \\
& \left\{\cos \left[m \Theta_{0 j}\left(p^{j}-k^{j}\right)\right]+\cos \left[m \Theta_{0 j}\left(p^{j}-k^{\prime j}\right)\right]\right\} .
\end{aligned}
$$

Notice that the magnetic part of the noncommutativity parameter $\left(\Theta_{i j}\right)$ does not contribute in the nonrelativistic limit.

From the above expressions, equivalent nonrelativistic potentials may be determined as follows. Usually these potentials are defined as the Fourier transform (FT) with respect to the transferred momentum $(\vec{k})$ of the scattering amplitudes. If the amplitudes depend only on $\vec{k}$ the corresponding (FT) will be local depending only on the relative coordinate $\vec{r}$. In our case the amplitudes depend not only on $\vec{k}$ but also on the initial momentum $\vec{p}$ of the scattered particle. Because of the noncommutativity of momentum and position operators, the construction of the potentials from these FT may be afflicted by ordering ambiguities. We solve these ambiguities by requiring hermiticity of the resulting expression. After that we check that the result is an effective potential in the sense that its momentum space matrix elements correctly reproduces the amplitudes that we had ab initio. Proceeding in this way, we are led to introduce

$$
\delta_{\epsilon_{1}^{\prime} \epsilon_{1}} \delta_{\epsilon_{2}^{\prime} \epsilon_{2}} \mathcal{M}^{F}(\vec{k}, \vec{p}) \equiv T_{a}^{L}(\vec{k}, \vec{p})+T_{c, \text { dir }}^{L}(\vec{k}, \vec{p})
$$

and

$$
\mathcal{M}^{B}(\vec{k}, \vec{p}) \equiv \bar{T}_{c, d i r}^{L}(\vec{k}, \vec{p}),
$$

for the fermionic and bosonic scattering amplitudes. The FT transform of the amplitudes are then given by

$$
V^{F, B}(\vec{r}, \vec{p})=(2 \pi)^{3} \int d^{3} k \mathcal{M}^{F, B}(\vec{k}, \vec{p}) e^{i \vec{k} \cdot \vec{r}}
$$

where the subscript dir is used to indicate that only the direct part of the amplitudes enter in the calculation. The corresponding quantum operators are obtained through the replacements $\vec{r} \rightarrow \vec{R}, \vec{p} \rightarrow \vec{P}$, where $\vec{R}$ and $\vec{P}$ are the Cartesian position and momentum operators obeying, by assumption, the canonical commutation relations $\left[R^{j}, R^{l}\right]=\left[P^{j}, P^{l}\right]=0$ and $\left[R^{j}, P^{l}\right]=i \delta^{j l}$. We have [18]

$$
\begin{aligned}
\hat{V}^{F}(\vec{R}, \vec{P}) & =-\left(\frac{g}{m}\right)^{2} \int \frac{d^{3} k}{(2 \pi)^{3}}\left(e^{i k^{l} R^{l}} e^{i k^{l} \Theta_{l j} P^{j}}+e^{i k^{l} R^{l}} e^{-i k^{l} \Theta_{l j} P^{j}}\right) \\
& -\frac{2}{3}\left(\frac{g}{m}\right)^{2}\left[\delta^{(3)}(\vec{R}+m \vec{\Theta})+\delta^{(3)}(\vec{R}-m \vec{\Theta})\right] \\
& +\frac{1}{3}\left(\frac{g}{m}\right)^{2}\left[\delta^{(3)}(\vec{R}-m \vec{\Theta}) e^{-2 i m \vec{\Theta} \cdot \vec{P}}+e^{2 i m \vec{\Theta} \cdot \vec{P}} \delta^{(3)}(\vec{R}-m \vec{\Theta})\right],
\end{aligned}
$$

and

$$
\begin{aligned}
\hat{V}^{B}(\vec{R}, \vec{P}) & =\frac{1}{6}\left(\frac{g}{m}\right)^{2}\left[\delta^{(3)}(\vec{R}+m \vec{\Theta})+\delta^{(3)}(\vec{R}-m \vec{\Theta})\right] \\
& +\frac{1}{6}\left(\frac{g}{m}\right)^{2}\left[\delta^{(3)}(\vec{R}-m \vec{\Theta}) e^{-2 i m \vec{\Theta} \cdot \vec{P}}+e^{2 i m \vec{\Theta} \cdot \vec{P}} \delta^{(3)}(\vec{R}-m \vec{\Theta})\right]
\end{aligned}
$$


where $\vec{\Theta} \equiv\left\{\Theta^{0 j}, j=1,2,3\right\}$. Notice that the magnetic components $\Theta_{i j}$ only contribute to the first line of (24). This contribution is free from ordering ambiguities because of

$$
\left[k^{l} R^{l}, k^{m} \Theta_{m j} P^{j}\right]=i k^{l} k^{m} \Theta_{m j} \delta^{l j}=0 .
$$

As claimed before, it has been checked that (24) and (25) correctly reproduces the scattering amplitudes we had before. From those forms of the potentials we can draw the following conclusions:

1. In the case of space/space noncommutativity fermions may be pictured as rods oriented perpendicular to the direction of the incoming momentum. By contrast, in this case, the bosonic particles are not affected by the noncommutativity.

2. In the case of time/space noncommutativity both fermions and bosons are affected. The nonlocality manifest through the fact that the scattering occurs only when the particles are at a distance of the order of $m|\Theta|$.

No vestige of breaking of unitarity was found in spite of the presence of scattered advanced waves. However, this picture will most certainly break down when loop contributions are taken into consideration.

\section{Acknowledgements}

This work was partially supported by Fundação de Amparo à Pesquisa do Estado de São Paulo (FAPESP) and Conselho Nacional de Desenvolvimento Científico e Tecnológico (CNPq).

\section{References}

[1] H. Snyder, Phys. Rev. 71, 38 (1947).

[2] S. Doplicher, K. Fredenhagen, and J. E. Roberts, "The Quantum Structure of Spacetime at the Planck Scale and Quantum Fields", Commun. Math. Phys. 172, 187 (1995)

[3] N. Seiberg and E. Witten, "String theory and noncommutative geometry," JHEP 9909, 032 (1999), hepth/9908142.

[4] M. R. Douglas and N. A. Nekrasov, "Noncommutative Field Theory", Rev. Mod. Phys. 73, 977 (2001), hepth/0106048.

[5] R. J. Szabo, "Quantum Field Theory on Noncommutative Spaces", hep-th/0109162.

[6] N. Seiberg, L. Susskind, and N. Toumbas, Space-time non-commutativity and causality, hep-th/0005015.
[7] J. Gomis, T. Mehen, Space-time NC field theories and unitarity, Nucl. Phys. B591, 265 (2000), hepth/0005129.

[8] T. Filk, "Divergences in a Field Theory on Quantum Space", Phys. Lett. B376, 53 (1996)

[9] C. P. Martin and D. Sanchez-Ruiz, "The one-loop UV divergent structure of U(1) Yang-Mills theory on NC $R^{4}$ ", Phys. Rev. Lett. 83, 476 (1999), hep-th/9903077.

[10] M. Hayakawa, "Perturbative analysis on infrared and ultraviolet aspects of NC QED on $R^{4}$ ", hepth/9912167; "Perturbative analysis on infrared aspects of NC QED on $R^{4 ",}$ Phys. Lett. B478, 394 (2000), hep-th/9912094.

[11] F. T. Brandt, A. Das, and J. Frenkel, "General Structure of the Photon Selfenergy in Noncommutative QED", hep-th/0112127.

[12] S. Minwalla, M. V. Raamsdonk, and N. Seiberg, "Noncommutative Perturbative Dynamics", JHEP 02, 020 (2000), hep-th/9912072.

[13] L. Griguolo and M. Pietroni, "Wilsonian Renormalization Group and the Noncommutative IR/UV Connection, JHEP 0105,032 2001, hep-th/0104217.

[14] G. Amelino-Camelia, L. Doplicher, Soon-keon Nam, and Yun-Seok Seo, "Phenomenology of Particle Production and Propagation in String Motivated Canonical Noncommutative Space-Time", hep-th/0109191.

[15] M. Gomes, "Renormalization in Noncommutative Field Theory". In: XI J. A. Swieca Summer School - Particles and Fields, Campos de Jordo, 2001.

[16] A. A. Bichl, J. M. Grimstrup, H. Grosse, L. Popp, M. Schweda, and R. Wulkenhaar, "The Superfield Formalism Applied to the Noncommutative Wess Zumino Model", JHEP 0010:046, 2000; hep-th/0007050.

[17] B. A. Campbell and K. Kaminsky, "Noncommutative Field Theory and Spontaneous Symmetry Breaking", Nucl. Phys. B581, 240(2000), hep-th/0003137; "Noncommutative Linear Sigma Models", Nucl. Phys. B606, 613 (2001), hep-th/0102022; F. J. Petriello, "The Higgs Mechanism in Noncommutative Gauge Theories, Nucl. Phys. B601, 169 (2001), hepth/0101109; F. Ruiz Ruiz, "UV/IR Mixing and the Goldstone Theorem in Noncommutative Field Theory", hep-th/0202011.

[18] H. O. Girotti, M. Gomes, A. Yu. Petrov, V. O. Rivelles, and A. J. da Silva, "The Low Energy Limit of the Noncommutative Wess-Zumino Model", hep-th/0101159.

[19] H. O. Girotti, M. Gomes, V. O. Rivelles, and A. J. da Silva, "A Consistent Noncommutative Field Theory: the Wess-Zumino Model", Nucl. Phys. B587, 299 (2000). 